\title{
Deep Brain Stimulation for Dystonia: Patient Selection, Surgical Tech- nique, and Programming
}

\author{
Ron L. Alterman*,1 and Michele Tagliati ${ }^{2}$ \\ ${ }^{I}$ Department of Neurosurgery and ${ }^{2}$ Neurology, Mount Sinai School of Medicine, New York, New York, USA
}

\begin{abstract}
Deep brain stimulation (DBS) at the internal globus pallidus (GPi) is currently approved for the treatment of primary generalized and segmental dystonia. Younger age at surgery, shorter disease duration, and absence of fixed skeletal deformities correlate with a better response to stimulation. Patients with cervical dystonia may also improve. As a group, patients with secondary dystonias respond less well to DBS than do patients with primary dystonia; however, patients with dystonia secondary to anoxic brain injury who have grossly intact basal ganglia anatomy, and patients with tardive dystonia may represent secondary dystonia sub-types for whom pallidal DBS is a viable option. In this submission we review the selection of dystonia patients for DBS surgery and provide details of our approach to surgery and device programming.
\end{abstract}

Keywords: Deep brain stimulation, dystonia, globus pallidus, tardive dystonia, DYT1, stereotactic surgery.

\section{INTRODUCTION}

Dystonia is a neurological disorder characterized by twisting repetitive movements that result in abnormal, often painful postures [1]. Different muscle groups may be involved to a variable extent and severity. Dystonia is not one disease; rather, it is a neurological manifestation of many pathological conditions, many of which are poorly characterized. The prevalence estimates for primary dystonia in the general population range from two to 50 cases per million for early onset dystonia and from 30 to 7320 cases per million for late onset dystonia; however, prevalence rates are significantly higher in some ethnic groups [2-4].

The limitations of medical therapy for dystonia led to the development of various surgical interventions that targeted both the peripheral and central nervous systems. Most were ablative in nature and yielded only modest improvements [5, 6]. The successful use of deep brain stimulation (DBS) for the treatment of medically refractory Parkinson's disease (PD), and, in particular, the observation that pallidal interventions improve 'off medication' parkinsonian dystonia [7], led to investigations of pallidal DBS for the treatment of primary dystonia. A decade later, DBS at the internal globus pallidus (GPi) has been established as the most effective treatment presently available for primary dystonia and one of the most successful applications of DBS technology yet described. In this article we detail our approach to the use of DBS for the treatment of dystonia, including patient selection, device implantation, and programming. This approach has evolved in the course of treating more than 90 dystonia patients over the last 10 years.

\footnotetext{
*Address correspondence to this author at the Department of Neurosurgery, One Gustave L. Levy Place, Box 1136, New York, New York 10029, USA; Tel: 212-241-0050; Fax: 212-410-0603;

E-mail: ron.alterman@mountsinai.org
}

\section{CLASSIFICATION OF DYSTONIA}

Dystonia may be classified in three ways: 1- by the anatomical distribution of the abnormal movements; 2- by the age at symptom onset (early vs. late); and 3- by the absence or presence of a specific underlying etiology (i.e. primary vs. secondary) [1]. Intermittent contractions that are limited to a single body region characterize focal dystonias such as writer's cramp or spasmodic torticollis. Segmental dystonia affects contiguous body parts (eg. neck and shoulder). Widespread involvement of the axial and limb musculature characterizes generalized dystonia. Patients with early symptom onset (i.e. age < 26) are more likely to have a heritable form of dystonia and are more likely to suffer generalized symptoms. Conversely, dystonia that begins in adulthood is more likely to remain focal $[1,3]$.

A dystonia is classified as primary or idiopathic when dystonia is the sole neurological finding and when no structural brain abnormality or specific toxic, metabolic, traumatic, or infectious etiology is identified. The heritable forms of dystonia are traditionally included in this group. At least 13 different mutations have now been associated with dystonia, each mutation occurring at a unique gene locus $[3$, 8]. The most common form of genetic dystonia results from a three base pair GAG deletion of the gene encoding the protein torsin A [4, 8]. This mutation, referred to as DYT1, is associated with a form of childhood onset dystonia formerly known as dystonia musculorum deformans. or Oppenheim's disease. DYT1-associated dystonia is inherited in an autosomal dominant pattern but with a penetrance of just 30$40 \%$, suggesting that additional genetic and/or epigenetic factors contribute to its phenotypic expression $[4,8]$.

When a structural brain abnormality, specific underlying etiology, or a neurological finding in addition to dystonia is identified, a dystonia is classified as secondary or symptomatic [1]. Symptomatic dystonia is more prevalent than primary dystonia and may arise from a variety of causes includ- 
ing static encephalopathy, stroke, traumatic brain injury, or any number of toxic, metabolic, or infectious insults to the central nervous system. Consequently, this is a heterogeneous patient population with highly varied pathophysiologies and responses to treatment.

\section{MEDICAL THERAPY FOR DYSTONIA}

In most cases, medical therapy for dystonia is limited to symptom control and is marginally effective [8]. Anticholinergic medications (e.g. trihexyphenidyl) are the mainstay of medical therapy but often yield only modest improvements and, at the high doses employed for dystonia, may cause significant side effects such as drowsiness, blurred vision and poor memory. Additional medications that may be employed include baclofen, benzodiazepines, and tetrabenazine. A minority of patients with symptomatic generalized dystonia will benefit from therapy targeted at the underlying disorder. In particular, children and adolescents with "clinically pure" dystonia of unknown etiology should be evaluated for Wilson's disease, and should undergo a trial of levodopa therapy, as a small percentage will have DopaResponsive Dystonia and will experience a profound and sustained response to this medication [8].

Targeted injections of botulinum toxin can alleviate focal or segmental dystonia but this intervention is impractical in patients with generalized symptoms [8, 9]. Moreover, some patients will not respond to botulinum toxin injections initially and up to $10 \%$ may develop resistance over time through the production of blocking antibodies [9]. Chronic intrathecal baclofen infusion via implantable pumps has been used with some success in dystonia [10]; however, we believe that in their present configurations, DBS presents significant advantages relative to intrathecal baclofen therapy including the potential for a broader anatomical response, and significantly less hardware maintenance. Nevertheless, intrathecal baclofen therapy remains an important intervention, particularly in the setting of mixed spastic/dystonic cerebral palsy.

\section{PATIENT SELECTION}

At our institution, both a stereotactic neurosurgeon (RLA) and a neurologist with expertise in the diagnosis and management of complex movement disorders (MT) evaluate every dystonia patient prior to recommending DBS surgery. The neurologist ensures that the diagnosis of dystonia is correct, that the dystonia is properly classified, and that all reasonable medical therapies have been tried. The neurologist also programs the DBS device post-operatively, monitors patient progress employing validated clinical rating scales, and prescribes changes to the medication regimen as needed.

In the United States only the Activa ${ }^{\mathrm{TM}}$ DBS system (Medtronic, Inc., Minneapolis, MN) is approved under a Humanitarian Device Exemption and only for the treatment of primary dystonia. The treatment of secondary dystonia is currently considered to be 'off-label' though active investigation is underway to identify secondary dystonia subpopulations that may also benefit from DBS [11]. Patients should not be offered surgery unless their symptoms are disabling and they have failed to respond to standard medical therapies (see above). Patients with cervical dystonia should also have tried botulinum toxin injections. A recent MRI of the brain should be obtained to exclude the presence of structural lesions, which would indicate a secondary dystonia. Patients with generalized dystonia of childhood onset should be tested for Wilson's disease and the DYT1 mutation, and should receive an adequate trial of levodopa. Patients who test positively for Wilson's disease should be treated accordingly. Of course, general medical contraindications to surgery should be ruled out.

\section{SURGICAL TECHNIQUE}

The DBS device is comprised of four primary components that are implanted in two stages (Fig. 1). During the first stage, the stimulating lead(s) is implanted into the GPi stereotactically and secured by means of an anchoring system that also covers the burr hole through which the lead is inserted. The remaining two components (i.e. the extension cable(s) and pulse generator(s) (PG)) are implanted during the second procedure, which may be performed on the same day or shortly thereafter. It is acceptable to implant DBS leads bilaterally during the same procedure. Dystonia patients are typically younger than patients with PD or ET and, in our experience, tolerate the bilateral frontal lobe penetrations without difficulty.

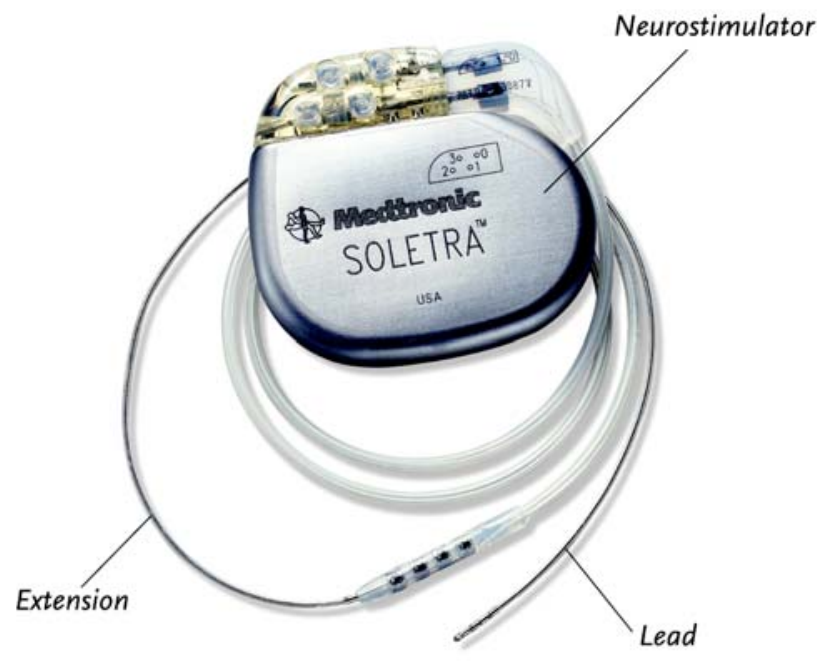

Fig. (1). The Deep Brain Stimulation System.

The DBS system (Activa ${ }^{\mathrm{TM}}$, Medtronic, Inc., Minneapolis, MN) has four primary components: 1 - the stimulating lead, which is implanted stereotactically into the desired target; 2 - the programmable neurostimulator, which generates the electrical impulses; 3- the extension cable, which is tunneled subcutaneously and connects the stimulator to the lead and; 4- the burr hole cap, which covers the burr hole and secures the lead in position (not pictured).

The lead implantation ideally is performed with the patient fully awake, but this may not be possible for younger children or patients with severely contorted postures. If painful muscular spasms or abnormal postures make awake surgery too onerous, conscious sedation with propofol and/or dexmedetomidine can be instituted. Anticholinergic medications and benzodiazepines are withheld on the morning of surgery as these medications may interfere with intraoperative microelectrode recording (MER). In the past we have also withheld baclofen but we now believe that baclofen withdrawal may have contributed to two episodes of post- 

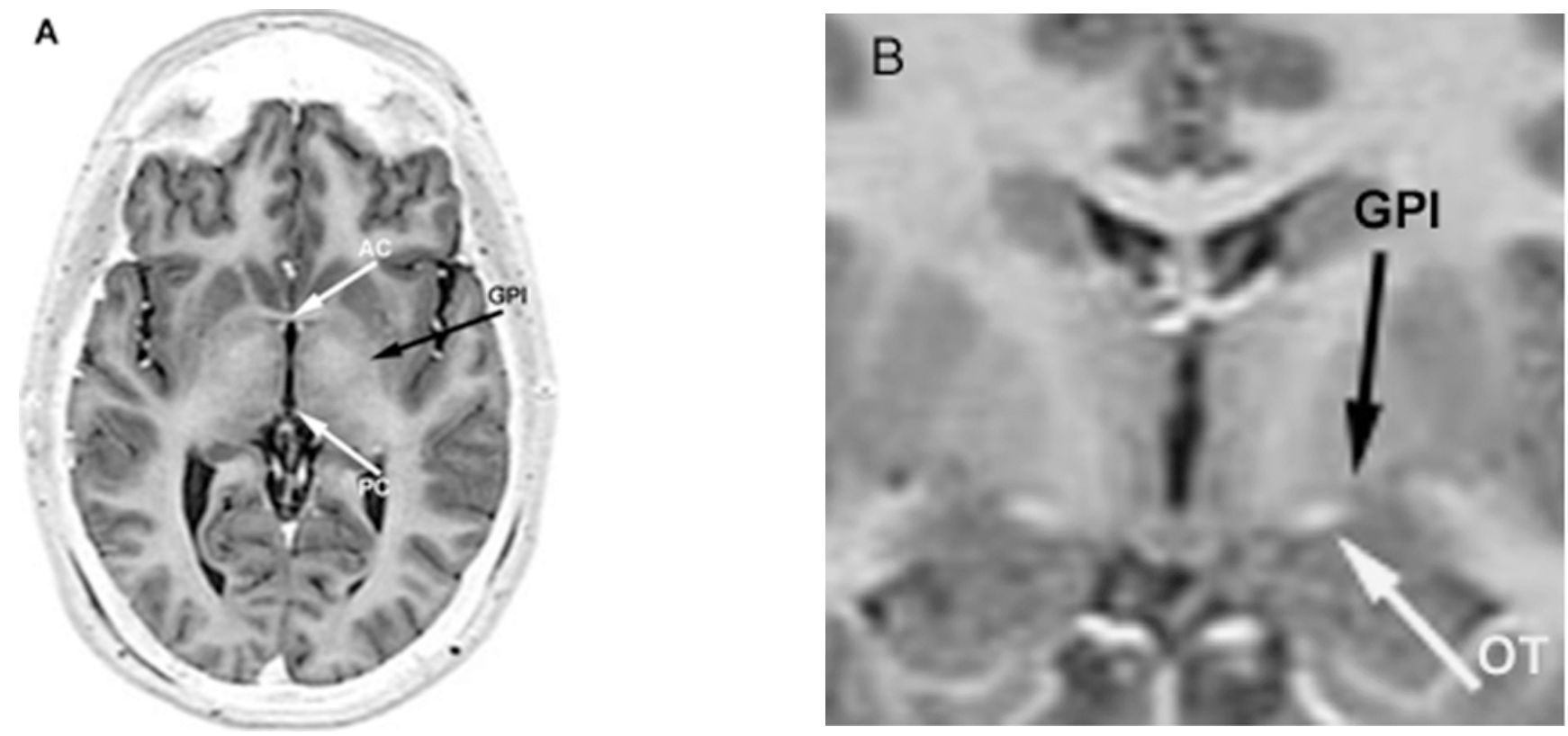

Fig. (2). Fast Spin Echo/Inversion Recovery MRI.

We employ both axial (A) and coronal (B) FSE/IR images for targeting the GPi. The anterior (Fig. 2A; black arrow) and posterior (Fig. 2A; white arrow) commissures are readily visible on the axial image, as is the posteroventral GPi. (Fig. 2A; black arrow). The target is the posteroventral GPi, approximately 20-21 mm lateral to the midline (Fig. 2B; black arrow), which lies) and 2-3 mm superior and lateral to the optic tract (Fig. 2B; white arrow).

operative dystonic crisis in patients who were taking particularly large doses of this medication. Consequently, we now advise patients to take their usual dose of baclofen on the morning of surgery.

A small dose of Fentanyl may be administered during application of the stereotactic frame (the most uncomfortable part of the whole procedure) but we avoid narcotics or benzodiazepines for the remainder of the operation. Administration of a total scalp block while the patient is sedated following stereotactic imaging also reduces patient discomfort and anxiety when they awaken. Antibiotics are administered intravenously during frame application so that serum levels are therapeutic during the implantation procedure.

\section{Anatomical Targeting}

Stereotactic headframes remain the 'gold standard' for performing DBS lead implants; however, "frame-less" technologies such as those manufactured by FHC Inc. (Bowdoinham, Maine, USA) and Medtronic, Inc. are being employed for DBS surgery with greater frequency [12]. We employ axial and coronal fast spin echo/inversion recovery (FSE/IR) MRI for anatomic targeting because the images are acquired rapidly (6-9 minutes per scan) and provide superior resolution of the commissures and deep nuclei (Fig. 2). Additionally, this pulse sequence is reported to resist distortion due to magnetic susceptibility artifact, minimizing the risk of targeting errors [13]. The thickness of the axial slices (2-3 $\mathrm{mm})$ required to generate these high resolution images increases our initial targeting error along the Z-axis (i.e. depth); but this is compensated for by microelectrode recording (MER), which delineates the position of specific structures along the implantation trajectory with a resolution of $0.1 \mathrm{~mm}$. The scanning parameters for FSE/IR MRI are given in Table 1. These images alone are sufficient for performing
DBS implants with microelectrode guidance; however, additional image sets such as gadolinium-enhanced threedimensionally acquired T1-weighted MRI (e.g. SPGR), and/or computerized tomography may also be employed. In particular, we have found that merging the FSE/IR images to gadolinium-enhanced volumetric T1 sequences (e.g. SPGR) improves the volume error of fiducial registration while providing a clear view of the cortical veins, which one is wise to avoid.

Table 1. Scanning Parameters for Fast Spin Echo/Inversion Recovery MRI

\begin{tabular}{|c|c|}
\hline Excitation Time (Te) & $120 \mathrm{msec}$ \\
\hline Relaxation Time (Tr) & $10,000 \mathrm{msec}$ \\
\hline Inversion Time (Ti) & $2200 \mathrm{msec}$ \\
\hline Band With & 20.83 \\
\hline Field of View (FOV) & 24 \\
\hline Slice Thickness & $3 \mathrm{~mm}$ \\
\hline Slice Spacing & $0 \mathrm{~mm}$ \\
\hline Frequency & $192 \mathrm{~Hz}$ \\
\hline Phase & 160 \\
\hline Number of Excitations & 1 \\
\hline Freqency Direction & Anteroposterior (AP) \\
\hline Autocontrol Frequency & Water \\
\hline Flow Compensation Direction & Slice Direction \\
\hline
\end{tabular}

Scanning parameters for fast spin echo/inversion recovery MRI are demonstrated. A scan of thirty slices can be obtained in 6-9 minutes employing these parameters. 
The stereotactic coordinates for the GPi may be calculated manually, employing the scanner's software. Alternatively, the imaging data sets may be uploaded to an independent workstation that is equipped with advanced stereotactic targeting software. These advanced software packages provide at least five distinct advantages: 1- the target coordinates are calculated automatically, eliminating human math errors; 2- a variety of image sets (e.g. CT and MRI) may be merged, allowing one to exploit the advantages of each; 3the entire trajectory may be visualized in all three anatomical planes of section and orthogonal to the trajectory (so-called "probes eye view"), allowing one to plan safer approaches to the target; 4- the imaging data sets are reformatted orthogonal to the intercommissural (IC) plane, thereby controlling for variations in headframe placement and; 5- digitized versions of stereotactic atlases may be overlaid and digitally "fit" to the patient's anatomy, helping to identify the desired target. A significant drawback to these systems is that they assume the patient's brain to be symmetric, which is not always the case. Cranial anomalies such as plagiocephaly can shift target structures and it is the surgeon's responsibility to account for these variables when targeting.

Currently, we target the internal pallidal site first described by Leksell and later popularized by Laitinen and colleagues [14], which lays 19-22 mm lateral, 2-3 mm anterior, and 4-5 $\mathrm{mm}$ inferior to the imaginary mid-commissural point (MCP). The target point is visualized on both axial and coronal FSE/IR images and should lay 2-3 $\mathrm{mm}$ superior and lateral to the optic tract (Fig. 2B). Our preferred trajectory approaches the target at angles of $60-65^{\circ}$ antero-superior to the intercommissural IC plane and $0-10^{\circ}$ lateral to the vertical axis. This virtually parasagittal trajectory avoids the ipsilateral lateral ventricle and facilitates the process of mapping the intra-operative microelectrode recording data onto human stereotactic atlases (see below).

\section{Microelectrode Recording}

We employ intra-operative single cell microelectrode recording (MER) to refine our anatomical targeting. The finer details of our MER technique are beyond the scope of this report but are provided elsewhere [15]. The need for MER is hotly debated; however, we find that MER provides important information that other neurophysiological localization techniques simply do not. First, MER delineates the borders and expanses of both the external and internal globus pallidus along a given trajectory with a spatial resolution of $\sim 100 \mu \mathrm{m}$. Second, the detection of kinesthetic cells confirms that the trajectory traverses the sensorimotor sub-region of the GPi, the target for the procedure. Third, delineating the inferior border of the GPi refines the depth of implantation, compensating for the 2-3 $\mathrm{mm}$ slice thickness of the axial targeting images. And fourth, identifying the optic tract 2-3 $\mathrm{mm}$ deep to the GPi exit point confirms that the trajectory exits the nucleus inferiorly, not posteriorly into the internal capsule.

The recording data are mapped onto scaled sagittal sections of human stereotactic atlases in order to determine the anatomical location of the recorded trajectory. Acceptable trajectories for implantation include a 3-4 $\mathrm{mm}$ span of external globus pallidus (GPe) and at least $7.5 \mathrm{~mm}$ of GPi. Such a trajectory must pass through the heart of the GPi and will allow three to four contacts to be positioned comfortably within the nucleus, depending on the lead employed (Fig. 3). Identification of the optic tract provides an additional level of confidence that the lead will be well-positioned; but this should not be viewed as an absolute requirement for implantation, as the optic tract may not always be identified.

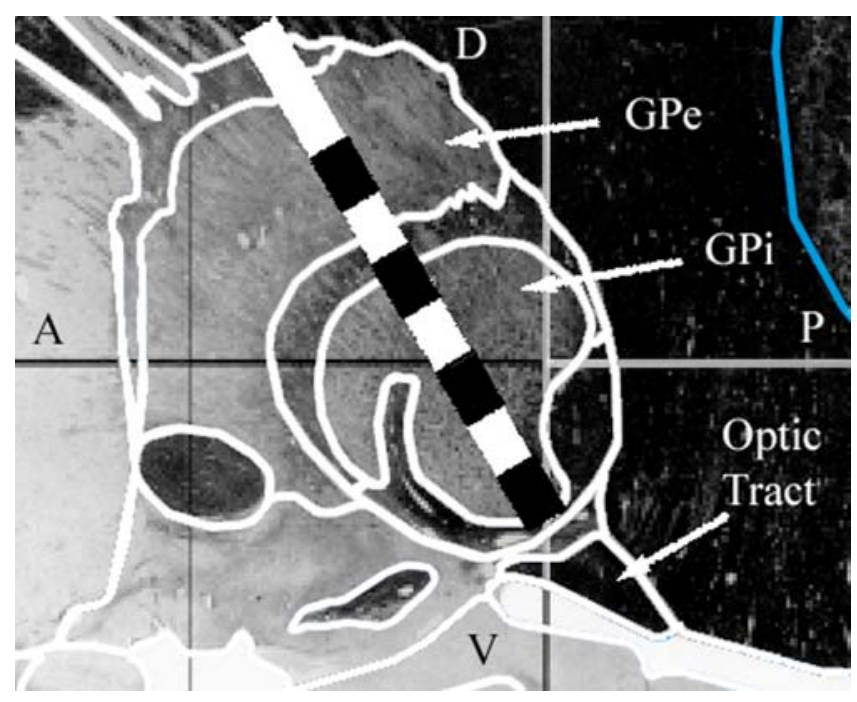

Fig. (3). Pallidal Lead Implantation.

Our preferred lead position within the GPi is depicted. (A) A schematic of the model 3387 lead (Medtronic Inc.), which has four 1.5 $\mathrm{mm}$ long cylindrical with $1.5 \mathrm{~mm}$ inter-electrode spacing, is superimposed on a sagittal image, $20 \mathrm{~mm}$ lateral of midline, derived from the Schaltenbrand and Wahren Atlas. With the deepest contact (contact 0) positioned at the inferior border of the GPi, three contacts can fit within the nucleus. Abbreviations: Globus Pallidus Externa (GPe); Globus Pallidus Interna (GPi); Anterior (A); Posterior (P); Dorsal (D); Ventral (V).

\section{Macroelectrode Stimulation}

The DBS lead is inserted along the desired trajectory leaving the deepest contact (contact 0 ) at the physiologically defined inferior border of the GPi (Fig. 3). C-arm fluoroscopy may be employed to confirm that the lead has traveled to the desired point, relative to the frame (Fig. 4). Before it is secured in place, the acute effects of stimulation via the lead are tested. Testing is performed in a bipolar configuration employing the following stimulation parameters: pulse width- $90 \mu \mathrm{sec}$; frequency- $130 \mathrm{~Hz}$, amplitude- 0-5V. Stimulation amplitudes greater than $5 \mathrm{~V}$ are not used as we have never required amplitudes this great for therapy. The initial test is performed with the deepest pair of contacts (i.e. 0-, $1+)$ as these are closest to the internal capsule and optic tract and are therefore most likely to generate adverse effects (AE). If no $\mathrm{AE}$ are observed, testing continues in a ventral to dorsal sequence (ie. 1-, 2+, etc.). Unlike Parkinson's disease, dystonia requires days to weeks of stimulation before improvements are apparent; therefore, a lack of improvement in response to intra-operative stimulation should not be viewed as an indicator of poor lead placement. If the microelectrode recording data is consistent with good placement and there are no AE observed with up to five volts of stimulation, the lead is very likely to be well positioned. 


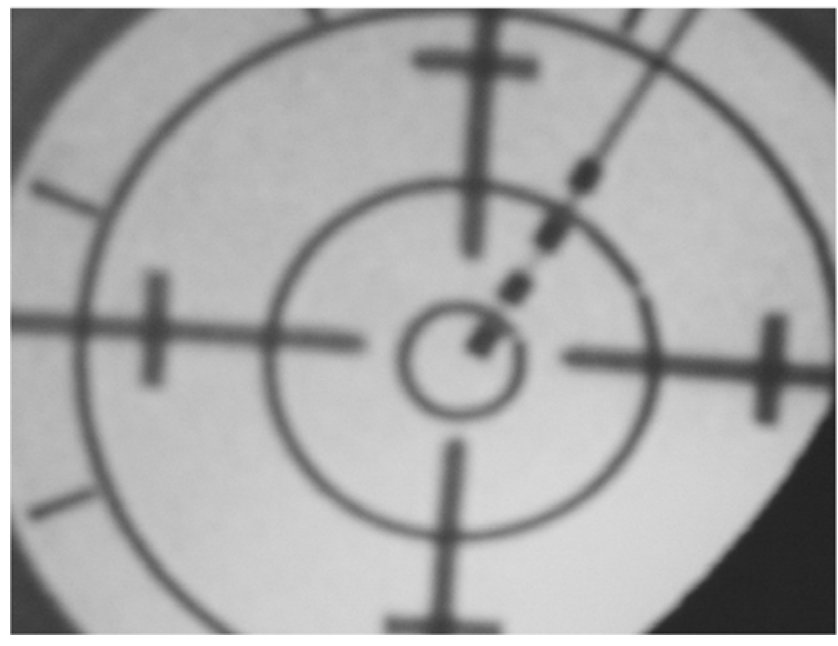

Fig. (4). Post-operative MRI.

A MRI is performed on all patients immediately after lead implantation image.

Sustained, time- and voltage-locked contractions of the contralateral hemi-body and/or face indicate that stimulation is activating the fibers of the corticospinal tract, in which case the lead is positioned too medially and/or posteriorly. The induction of phosphenes in the contralateral visual field suggests that stimulation is activating the optic tract and that the lead is too deep. Stimulation within the sensorimotor GPi will induce transient paresthesiae; however, sustained paresthesiae at low stimulation amplitudes indicate that the lead is positioned too posteriorly, and is activating thalamo-cortical projections in the posterior limb of the internal capsule. If any of these adverse effects occur, the lead should be repositioned accordingly.

The lead is secured at the skull employing a locking device that also covers the burr hole. Serial fluoroscopy is used to confirm that the lead is not displaced from its desired position during fixation. The free end of the lead is encircled around the burr hole cap and left in the sub-galeal space. The incision is irrigated with antibiotic containing saline and closed in a standard fashion. After removing the stereotactic frame, a post-operative MRI is performed to confirm that the leads are well-positioned and that there has been no hemorrhage (Fig. 5). Patients are observed overnight in the neurosurgical intensive care unit and discharged the following day.

\section{Implantation of the Pulse Generator}

The remaining components of the DBS system(s) are implanted under general anesthesia, usually within two weeks of the lead implants. This relatively simple procedure involves the following steps: 1- creating an infra-clavicular, subcutaneous pocket for the IPG; 2-identifying the free end of the DBS lead in the subgaleal space; 3- tunneling the extension cable subcutaneously from the IPG pocket to the free end of the DBS lead and; 4- establishing dry, clean, and secure connections between the components. The connection between the lead and the extension cable is placed under the galea, just lateral to the cranial incision, limiting exposure of the lead to potential fracture through movement. In very young patients with thin skin one may place the IPG(s) at the abdomen.

\section{PROGRAMMING THE DEVICE}

The device(s) is activated 2-4 weeks after lead implantation, allowing time for the surgical incisions to heal. The clinician controls five stimulus parameters: amplitude, pulse width, frequency, stimulus configuration (i.e. uni- vs. bipolar) and the active contact(s). There is no consensus regarding the optimal settings for treating dystonia as systematic evaluations of varying stimulus parameters have not been conducted. Instead, therapy is currently guided by published case series, which mainly report positive responses with wide pulses $(210-400 \mu \mathrm{sec})$ and high frequencies $(130 \mathrm{~Hz}$ or higher) [16-20]. Though effective, these parameters rapidly deplete the IPGs, necessitating their frequent replacement (12-24 months). We have found that stimulation at lower frequencies $(60-80 \mathrm{~Hz})$ and narrower pulses $(120-210 \mu \mathrm{sec})$ may be just as effective as high frequency stimulation [21, 22]. These settings deliver less electrical energy to the brain, enhancing the tolerability of stimulation and prolonging battery life [23].

At the initial programming session, the effects of unipolar stimulation with each of the contacts in isolation are assessed with particular attention paid to the stimulation thresholds for inducing AE. For therapy, we employ the ventral-most contact(s) as tolerated. The wisdom of this approach is supported by studies demonstrating that activation of the most posteroventral GPi contacts correlated with superior outcomes in dystonia patients treated with pallidal DBS $[24,25]$. We prefer to treat with unipolar stimulation but will use bipolar settings if unipolar stimulation is not tolerated. Patients are initially treated at $2.0-2.5 \mathrm{~V}$. The stimulation amplitude may be increased over time; however, every effort should be made not to exceed 3.6 V if Soletra (Medtronic) neurostimulators are used, as the IPG must invoke a 'doubling circuit' to deliver amplitudes above this threshold, shortening battery life out of proportion to the energy delivered. If more energy is required, it is preferable to increase the frequency or pulse duration from the standpoint of battery preservation.

Patients return every two-to-four weeks for evaluation during the first three months, and every three to six months after that. During each visit the patient is assessed employing a variety of standardized clinical rating scales including the Burke-Fahn-Marsden Dystonia Rating Scale (BFMDRS) [1, 26].

\section{CLINICAL RESULTS}

The efficacy of pallidal DBS for primary generalized or segmental dystonia has been well documented in both retrospective case series and prospective clinical trials [16-34]. These studies consistently demonstrate that pallidal DBS yields significant motor and clinical benefit in patients with primary generalized or segmental dystonia, as measured with the Burke-Fahn- Marsden Dystonia Rating Scale (BFMDRS). According to a recent review, at least 249 individual cases of pallidal DBS for primary dystonia have now been reported in the literature [33]. Most are reported as part of small, open-label case series and most authors report improvements in the BFMDRS of $60-70 \%$.

In a retrospective analysis of 39 consecutive primary dystonia patients, we found that patients with fixed skeletal 
deformities (FSD) at the time of surgery experienced a more limited improvement than patients without FSD [34]. Among the patients without FSD, we found that younger age $(<20$ years) and shorter disease duration ( $<15$ years) at the time of surgery was associated with a superior response to DBS at one year. No other factor, including presence of the DYT1 gene mutation was predictive of outcome.

Tisch et al. [24] examined the relationship of clinical outcome and lead position relative to the intercommissural plane and found that leads positioned more posteriorly and ventrally within the GPi yielded greater BFMDRS motor score improvements than leads positioned more anteriorly and dorsally. These results confirm and expand upon prior results $[35,36]$ and suggest that Leksell's posteroventral GPi target remains the optimal site within this nucleus for treating dystonia whether by stimulation or ablation.

The data supporting the use of pallidal DBS for Cervical Dystonia (CD) is not yet as developed as that available for PGD. Preliminary reports of small case series suggest that $\mathrm{CD}$ is responsive to bilateral pallidal DBS, with improvements in the Toronto Western Spasmodic Torticollis Rating Scale (TWSTRS) ranging from 43-76\% [37-39]. The only multi-center, prospective, single-blind trial of pallidal DBS in $10 \mathrm{CD}$ patients reported significant improvements in the TWSTRS severity, disability, and pain sub-scales as well as reduced symptoms of depression and enhanced quality of life measures [38]. Overall, the response of CD to pallidal DBS appears to be less consistent than that observed in PGD; however, larger and longer-term analyses of DBS in CD are necessary to document accurately the response to DBS.

\section{Pallidal DBS for Secondary Dystonia}

Thus far, pallidal DBS has proven far less effective in secondary dystonia than in PGD [40-42] and consequently, DBS for secondary dystonia is still considered to be an 'offlabel' indication. Patients with secondary dystonia represent a heterogeneous population with regard to etiology, clinical signs, and long-term prognosis. Many of them have other neurological disorders in addition to dystonia (e.g. seizures, spastic paresis, cerebellar and brainstem dysfunction, and developmental delay) that limit their functional response to DBS, which is directed solely at their dystonia. Nevertheless, evidence is emerging that some sub-populations of secondary dystonia patients may respond well enough to DBS to warrant the risks of surgery. These include patients with perinatal hypoxic brain injuries $[11,43]$ and tardive dystonia [44-47].

\section{CONCLUSIONS}

Deep brain stimulation at the internal globus pallidus has emerged as the treatment of choice for medically refractory primary torsion dystonia. Multiple open-label studies demonstrate that pallidal DBS is highly effective in patients with PGD and is well tolerated. Children and patients who are DYT1-positive may fare best of all. Preliminary results in patients with spasmodic torticollis are promising but larger case series are required before the true efficacy of pallidal DBS for this entity can be ascertained. As a whole, patients with secondary dystonia respond more modestly and inconsistently than do primary dystonia patients, reflecting the physiologic and anatomic heterogeneity of this population.
Those with tardive dystonia and dystonia secondary to anoxic brain injury, but with preserved basal ganglia anatomy, represent sub-groups that may respond well to DBS therapy.

\section{ACKNOWLEDGEMENTS}

The author wishes to thank Donald Weisz, PhD for his assistance with the production of Fig. (3).

\section{REFERENCES}

[1] Fahn S. Idiopathic torsion dystonia In: Calne DB, Ed. Neurodegenerative diseases. Philadelphia: W. B. Saunders, 1994; pp. 705-15.

[2] Defazio G, Abbruzzese G, Livrea P, Berardelli A. Epidemiology of primary dystonia. Lancet Neurol 2004; 3: 673-8.

[3] Bressman SB. Dystonia: phenotypes and genotypes. Rev Neurol (Paris) 2003; 159: 849-56.

[4] Bressman SB, Sabatti C, Raymond D, et al. The DYT1 phenotype and guidelines for diagnostic testing. Neurology 2000; 54: 1746-52.

[5] Krack P, Vercueil L. Review of the functional surgical treatment of dystonia. J Eur Neurol 2001; 8: 389-99.

[6] Braun V, Richter HP. Selective peripheral denervation for spasmodic torticollis: 13-year experience with 155 patients. J Neurosurg 2002; 97: 207-12.

[7] Lozano AM, Lang AE, Galvez-Jimenez N, et al. Effect of GPi pallidotomy on motor function in Parkinson's disease. Lancet 1995; 346: 1383-7.

[8] Tagliati M, Golden A, Bressman S. Childhood dystonia. In Watts RL, Koller WC, Eds. Movement disorders: neurologic Principles and practice. New York: McGraw Hill, 2004; pp. 495-510.

[9] Greene P, Fahn S, Diamond B. Development of resistance to botulinum toxin type A in patients with torticollis. Mov Disord 1994; 9: 213-7.

[10] Ford B, Greene P, Louis ED, et al. Use of intrathecal baclofen in the treatment of patients with dystonia. Arch Neurol 1996; 53: 1241-6.

[11] Vidailhet M, Yelnik J, LaGrange C, et al. Bilateral pallidal deep brain stimulation for the treatment of patients with dystoniachoreoathetosis cerebral palsy: a prospective pilot study. Lancet Neurol 2009; 8(8): 709-17.

[12] Henderson JM, Holloway KL. Achieving optimal accuracy in frameless functional neurosurgical procedures. Stereotact Funct Neurosurg 2008; 86(5): 332-3.

[13] Taren JA, Ross DA, Gebarski SS. Stereotactic localization using fast spin-echo imaging in functional disorders. Acta Neurochir Suppl (Wien) 1993; 58: 59-60.

[14] Laitinen LV, Bergenheim AT, Hariz MI. Leksell's posteroventral pallidotomy in the treatment of Parkinson's disease. J Neurosurg 1992; 76: 53-61.

[15] Shils J, Tagliati M, Alterman R. Neurophysiological monitoring during neurosurgery for movement disorders. In Deletis V, Shils J, Eds. Neurophysiology in neurosurgery. San Diego: Academic Press, 2002; pp. 393-436.

[16] Coubes P, Echenne B, Roubertie A, et al. Treatment of early-onset generalized dystonia by chronic bilateral stimulation of the internal globus pallidus. Apropos of a case. Neurochirurgie 1999; 45: 13944.

[17] Krauss JK, Loher TJ, Weigel R, et al. Chronic stimulation of the globus pallidus internus for treatment of non-dYT1 generalized dystonia and choreoathetosis: 2-year follow-up. J Neurosurg 2003; 98: 785-92.

[18] Yianni J, Bain P, Giladi N, et al. Globus pallidus internus deep brain stimulation for dystonic conditions: a prospective audit. Mov Disord 2003; 18(4): 436-42.

[19] Coubes P, Cif L, El Fertit H, et al. Electrical stimulation of the globus pallidus internus in patients with primary generalized dystonia: long-term results. J Neurosurg 2004; 101: 189-94.

[20] Vidailhet M, Vercueil L, Houeto JL, et al. Bilateral deep brain stimulation of the globus pallidus in primary generalized dystonia. N Engl J Med 2005; 352: 459-67.

[21] Alterman R, Shils J, Miravite J, et al. A lower stimulation frequency can enhance tolerability and efficacy of pallidal deep brain stimulation for dystonia. Mov Disord 2007; 22: 366-8.

[22] Alterman R, Miravite J, Shils J, et al. 60 Hertz Pallidal deep brain stimulation for primary torsion dystonia. Neurology 2007; 69: 6818. 
[23] Isaias IU, Alterman RL, Tagliati M. Deep brain stimulation for primary dystonia: long-term outcomes. Arch Neurol 2009; 66(4): 465-70.

[24] Tisch S, Zrinzo L, Limousin P, et al. Effect of electrode contact location on clinical efficacy of pallidal deep brain stimulation in primary generalised dystonia. J Neurol Neurosurg Psychiatry 2007; 78(12): 1314-9.

[25] Houeto JL, Yelnik J, Bardinet E, et al. Acute deep-brain stimulation of the internal and external globus pallidus in primary dystonia: functional mapping of the pallidum. Arch Neurol 2007; 64(9): 1281-6.

[26] Volkmann J, Benecke R. Deep brain stimulation for dystonia: patient selection and evaluation. Mov Disord 2002; 17(Suppl 3): S112-5.

[27] Kumar R, Dagher A, Hutchison WD, et al. Globus pallidus deep brain stimulation for generalized dystonia: clinical and PET investigation. Neurology 1999; 53: 871-4.

[28] Vercueil L, Pollak P, Fraix V, et al. Deep brain stimulation in the treatment of severe dystonia. J Neurol 2001; 248: 695-700.

[29] Kupsch A, Kuehn A, Klaffke S, et al. Deep brain stimulation in dystonia. J Neurol 2003; 250(Suppl 1): 47-52.

[30] Yianni J, Bain PG, Gregory RP, et al. Post-operative progress of dystonia patients following globus pallidus internus deep brain stimulation. Eur J Neurol 2003; 10: 239-47.

[31] Kupsch A, Benecke R, Muller J, et al. Pallidal deep-brain stimulation in primary generalized or segmental dystonia. N Engl J Med 2006; 355: 1978-90

[32] Vidailhet M, Vercueil L, Houeto JL, et al. Bilateral, pallidal, deepbrain stimulation in primary generalised dystonia: a prospective 3 year follow-up study. Lancet Neurol 2007; 6(3): 223-9.

[33] Ostrem JL, Starr PA. Treatment of dystonia with deep brain stimulation. Neurotherapeutics 2008; 5: 320-30.

[34] Isaias IU, Alterman RL, Tagliati M. Outcome predictors of pallidal stimulation in patients with primary dystonia: the role of disease duration. Brain 2008; 131: 1895-902.

[35] Starr PA, Turner RS, Rau G, et al. Microelectrode-guided implantation of deep brain stimulators into the globus pallidus internus for dystonia: techniques, electrode locations, and outcomes Neurosurg Focus 2004; 17: E4

[36] Hamani C, Moro E, Zadikoff C, et al. Location of active contacts in patients with primary dystonia treated with globus pallidus deep brain stimulation. Neurosurgery 2008; 62: 217-23.

[37] Bittar RG, Yianni J, Wang SY, et al. Deep brain stimulation for generalized dystonia and spasmodic torticollis. J Clin Neurosci 2005; 12: 12-6.

[38] Kiss ZHT, Doig-Beyaert K, Eliasziw M, et al. The Canadian multicentre study of deep brain stimulation for cervical dystonia. Brain 2007; 130: 2879-86.

[39] Hung SW, Hamani C, Lozano AM, et al. Long-term outcome of bilateral pallidal deep brain stimulation for primary cervical dystonia. Neurology 2007; 68: 457-9.

[40] Elthaway H, Saint-Cyr J, Giladi N, Lang A, Lozano AM. Primary dystonia is more responsive than secondary dystonia to pallidal interventions: outcome after pallidotomy or pallidal deep brain stimulation. Neurosurgery 2004; 54: 613-21.

[41] Alterman, RL, Snyder BJ. Deep brain stimulation for torsion dystonia. Acta Neurochirurgica 2007; 97(2): 191-9.

[42] Zhang J, Zhang K, Wang Z, et al. Deep brain stimulation in the treatment of secondary dystonia. Chin Med J 2006; 119: 2069-74.

[43] Zorzi G, Marras C, Nardocci N, et al. Stimulation of the globus pallidus internus for childhood-onset dystonia. Mov Disord 2005; 20: 1194-2000.

[44] Franzini A, Marras C, Ferroli P, et al. Long-term high-frequency bilateral pallidal stimulation for neuroleptic-induced tardive dystonia. Report of two cases J Neurosurg 2005; 102: 721-5.

[45] Trottenberg T, Volkmann J, Deuschl G, et al. Treatment of severe tardive dystonia with pallidal deep brain stimulation. Neurology 2005; 64(2): 344-6.

[46] Sako W, Goto S, Shimazu H, et al. Bilateral deep brain stimulation of the globus pallidus internus in tardive dystonia. Mov Disord 2008; 23: 1929-31.

[47] Gruber D, Trottenberg T, Kivi A, et al. Long-term effects of pallidal deep brain stimulation in tardive dystonia. Neurology 2009; 73(1): 53-8.

(C) Alterman and Tagliati; Licensee Bentham Open.

This is an open access article licensed under the terms of the Creative Commons Attribution Non-Commercial License (http://creativecommons.org/licenses/by-nc/3.0/) which permits unrestricted, non-commercial use, distribution and reproduction in any medium, provided the work is properly cited. 